\title{
Bullous pyoderma gangrenosum associated with pancytopenia of unknown origin
}

\author{
Katarzyna Wolska', Małgorzata Michalska-Jakubus², Joanna Pucuła², Grażyna Chodorowska², Grzegorz Dzida³ \\ Jerzy Mosiewicz ${ }^{3}$, Andrzej Prystupa ${ }^{3}$, Monika Podhorecka ${ }^{4}$, Dorota Krasowska ${ }^{1,2}$
}

\author{
${ }^{1}$ Medical Students' Research Association at Department of Dermatology, Venereology and Paediatric Dermatology, Medical University \\ of Lublin, Poland \\ Scientific supervisor: Prof. Dorota Krasowska MD, PhD \\ ${ }^{2}$ Department of Dermatology, Venereology and Paediatric Dermatology, Medical University of Lublin, Poland \\ Head of Department: Prof. Grażyna Chodorowska MD, PhD \\ Department of Internal Medicine, Medical University of Lublin, Poland \\ Head of Department: Prof. Jerzy Mosiewicz MD, PhD \\ ${ }^{4}$ Department of Haematology-Oncology, Medical University of Lublin, Poland \\ Head of Department: Prof. Anna Dmoszyńska MD, PhD
}

Postep Derm Alergol 2014; XXXI, 4: 272-276

DOI: $10.5114 /$ pdia.2014.40980

\begin{abstract}
Pyoderma gangrenosum (PG) is a neutrophilic dermatosis of unknown origin. Clinically it starts with a pustule, nodule or bulla that rapidly progresses and turns into a painful ulcer with raised, undermined borders. The etiopathogenesis of PG remains unknown. However it is frequently associated with systemic diseases such as inflammatory bowel disease (IBD), haematological disorders or arthritis. The latest multicentric retrospective analysis published by Ghazal et al. shows that anaemia has been observed very often in German patients suffering from PG (in $45.6 \%$ of 259) so this disorder is supposed to be a possible cofactor in the pathogenesis of PG. According to its progressive course, patients require intensive diagnostic procedures and rapid initiation of the treatment. In this article, we report a case of bullous pyoderma gangrenosum in association with pancytopenia of unknown origin, according to its diagnostic and therapeutic difficulties.
\end{abstract}

Key words: bullous pyoderma gangrenosum, pancytopenia.

\section{Introduction}

Pyoderma gangrenosum (PG) is a rare, chronic neutrophilic dermatosis that presents as progressive necrosis of the skin [1]. Pyoderma gangrenosum was first described and named by Brunsing et al. in 1930 [2]. They erroneously believed that streptococcal infection was a significant component leading to secondary cutaneous gangrene and named the disease pyoderma gangrenosum. Although the precise etiopathogenesis is not fully understood, immunological mechanisms are considered to be involved. The development of lesions in the areas of incidental or iatrogenic trauma (pathergy phenomenon) may also play some role in its pathogenesis [2]. Clinically PG starts with a sterile pustule, nodule or bulla that rapidly progresses and turns into painful ulceration with raised, undermined borders and necrotic eschar [3]. Pyoderma gangrenosum occurs most commonly on the lower limbs with preference for the pretibial area (60\% of cases). However, upper limbs, the thorax and face can also be affected. More than $50 \%$ of patients with PG have an associated systemic disease, most frequently inflammatory bowel disease, haematological disorders, or arthritis. Incidence peaks between the ages of 20 and 50 years, with women more commonly affected. The disease is rare, with an estimated incidence of 3 to 10 cases per million people per year. Pyoderma gangrenosum in infants accounts for only $4 \%$ of cases and the disease has occasionally been reported in the elderly. Pyoderma gangrenosum is diagnosed based on distinctive clinical features and exclusion of other diseases with a similar appearance. Although the histopathologic findings are not diagnostic, a skin biopsy is necessary to rule out other causes of skin ulceration [4].

Address for correspondence: Prof. Dorota Krasowska MD, PhD, Department of Dermatology, Venereology and Paediatric Dermatology, Medical University of Lublin, 13 Radziwitłowska St, 20-080 Lublin, Poland, phone/fax: +48 8153236 47,

e-mail: dorota.krasowska@umlub.pl

Received: 9.12.2013, accepted: 25.01.2014. 
We report a case of bullous PG in association with pancytopenia of unknown origin.

\section{Case report}

A 56-year-old woman was admitted to the Department of Dermatology (September 2011) due to infiltrative erythematous lesions with seropurulent exudation and crusting, located on the right cheek, temple, and eyelid, treated with acyclovir since ophthalmic zoster was suspected (Figure $1 \mathrm{~A}$ ). A few days later, the patient became febrile up to $39^{\circ} \mathrm{C}$ and the lesions spread to the analogous left-sided area and were recognized by the ophthalmologist as preseptal cellulitis. In 2002, the woman was diagnosed with breast cancer, and underwent a left mastectomy as well as chemotherapy. Since 2008, the patient has been under the supervision of the Haematology-Oncology Clinic for leucopenia. She had no history of other symptoms and diseases. On admission, laboratory results were significant for severe anaemia and thrombocytopenia (HGB $6.8 \mathrm{~g} / \mathrm{dl}$, RBC $2.02 \mathrm{M} / \mu \mathrm{l}$, HCT 20.6\%, PLT $49 \mathrm{~K} / \mu \mathrm{l}$ ), in addition to the elevated erythrocyte sedimentation rate (ESR $130 \mathrm{~mm} / \mathrm{h}$ ) and C-reactive protein level (CRP 219.3 $\mathrm{mg} / \mathrm{l})$. Immunofixation electrophoresis revealed raised levels of $\alpha_{1}$-globuline (7\%; normal 3-5\%) and $\alpha_{2}$-globuline (18.4\%; normal 7-13\%), whereas $\beta$-globulin, $\gamma$-globulin and $A / G$ index were within normal limits. Immunological investigations were negative for antineutrophil cytoplasmic antibodies (pANCA, cANCA). Cultures of skin lesions and blood were negative for bacterial infection. Empiric antibiotic therapy (Cefuroxime), intravenous immunoglobulin infusions, packed red blood cells (PRBC) and local therapy were introduced, which reduced periorbital inflammation and infiltration. However, new oedematous lesions with erythema and infiltrate developed in the right cubital fossa and left supraclavicular region, accompanied by hemorrhagic bullae with necrosis on the right pretibial area and dorsal aspect of the left hand (Figure $1 \mathrm{~B}$ ). Due to progressing anaemia and thrombocytopenia, the patient was referred to the Department of Internal Medicine for further examinations. Ultrasound and computed tomography (CT) of the abdominal cavity did not reveal any pathology except for enlarged spleen (126 mm). Other examinations (gastroscopy, chest X-ray, ECG) were within normal limits. Trephine biopsy showed normocellular bone marrow with normal maturation and no features of dysplasia or neoplastic infiltrate. A skin biopsy taken from the edge of an ulcer demonstrated a dense neutrophilic
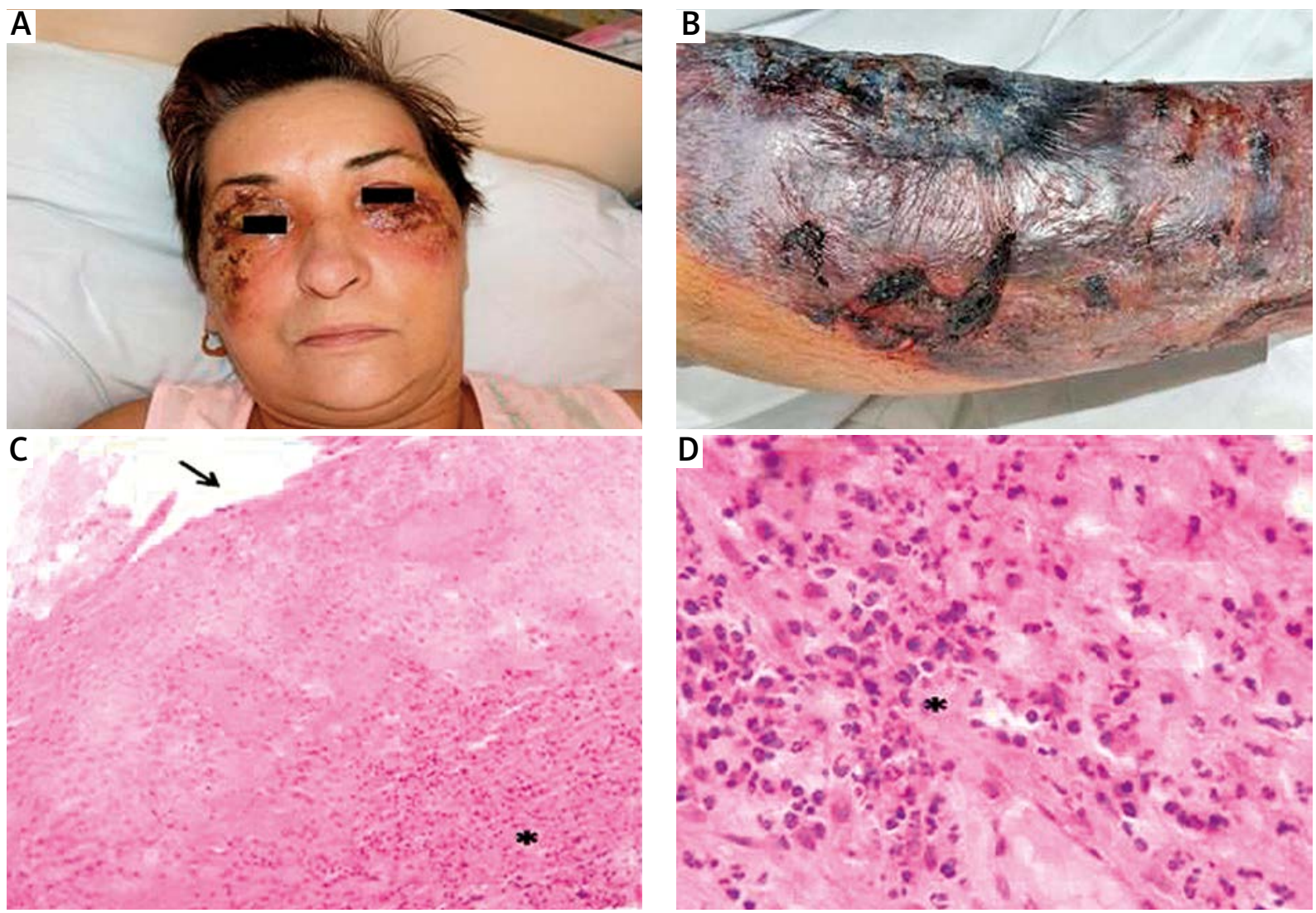

Figure 1. Infiltrative, erythematous lesions with seropurulent exudation and crusting on the face (A) and haemorrhagic bullae with necrosis on the right pretibial area (September/October 2011) (B); histopathology (H + E) from the edge of hemorrhagic bullae; dense neutrophilic and perivascular lymphocytic infiltrate $\left.{ }^{*}\right)$; epidermal detachment $(\downarrow)$ (C, D) 
and perivascular lymphocytic infiltrate, consistent with neutrophilic dermatosis (Figures 1 C-D). Based on medical history, distinctive clinical features, exclusion of other ulcerative diseases and additionally on histopathologic findings, the diagnosis of PG was established. The initial therapy with an intravenous pulse of methylprednisolone ( $4 \times$ $250 \mathrm{mg}$ ) and a course of prednisone (30 mg/day) resulted in partial resolution of hemorrhagic bullae as well as normalization of body temperature, ESR and CRP without improvement in blood cell count. Malar and periorbital lesions resolved completely. However, with tapering of the prednisone dose, there was a relapse of PG in December 2011 - lesions on the pretibial area reoccurred with a concomitant increase in the ESR $(90 \mathrm{~mm} / \mathrm{h})$ and CRP levels (210 mg/l). Therefore, a second infusion of methylprednisolone was administered and cyclosporine A (CsA; 200 mg/
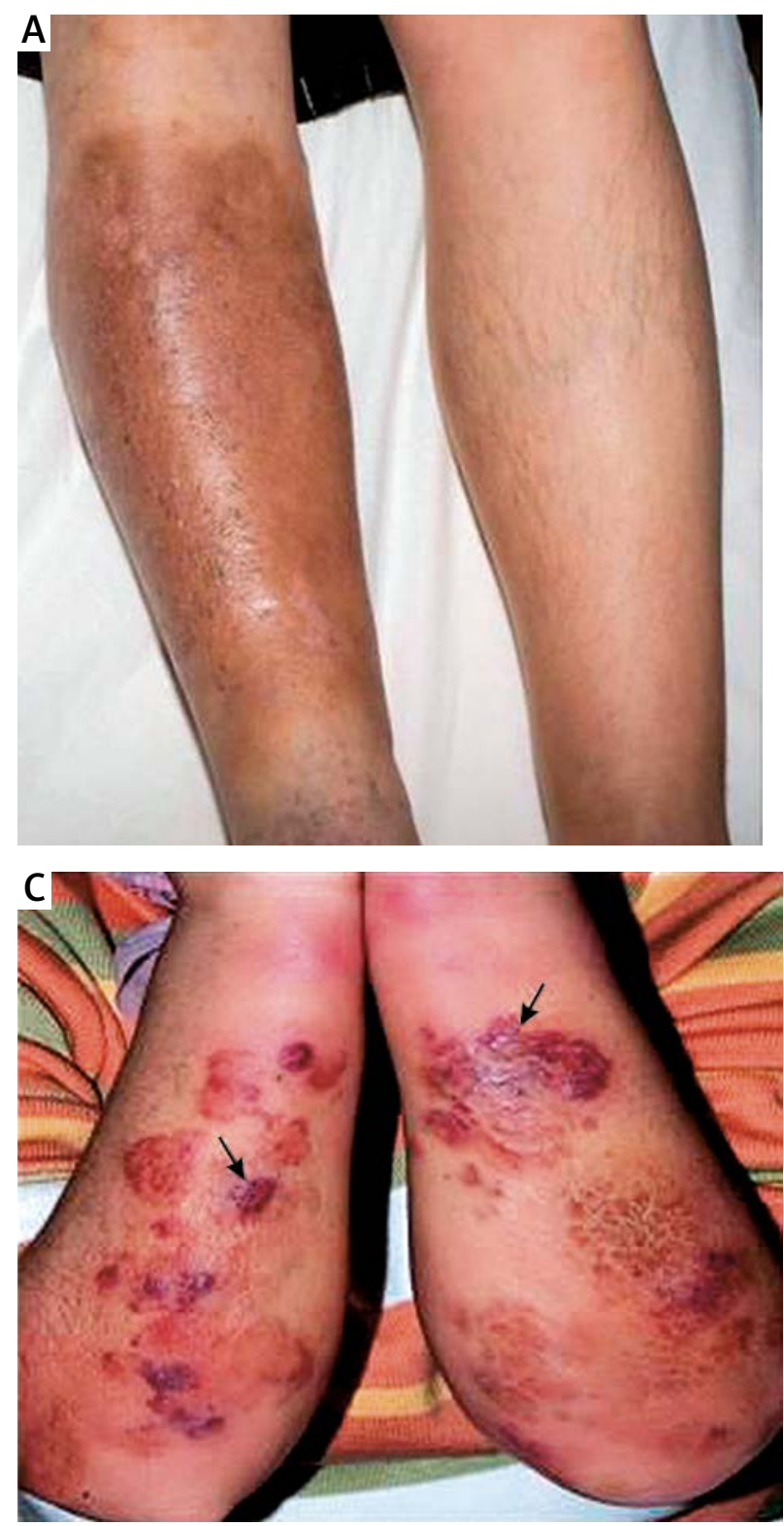

Figure 2. Clearance of facial and pretibial lesions after pulsed methylprednisolone and CsA (200 mg/day) (A, B); erythemato-oedematous lesions with hemorrhagic bullae on the forearms after tapering down CsA on may 2012 (C)

day) was introduced, resulting in a complete clearance of skin lesions (Figures 2 A-B). In May 2012, tapering down CsA to $150 \mathrm{mg} /$ day led to the formation of new erythemato-oedematous lesions with hemorrhagic bullae on the forearms (Figure 2 C). The third pulse of methylprednisolone was administered and the dose of CSA was increased to $200 \mathrm{mg} /$ day with good response. However, blood cell count was indicative of progressing anaemia and thrombocytopenia with slight leucopenia and the patient was hospitalized several times at the Department of Internal Medicine and the Department of Haematology-Oncology. The subsequent trephine biopsies did not reveal any acute proliferation of the haematopoietic system, however slight features of myelodysplasia did occur. Due to the history of breast cancer, a positron emission tomography scan was performed and gave normal results.
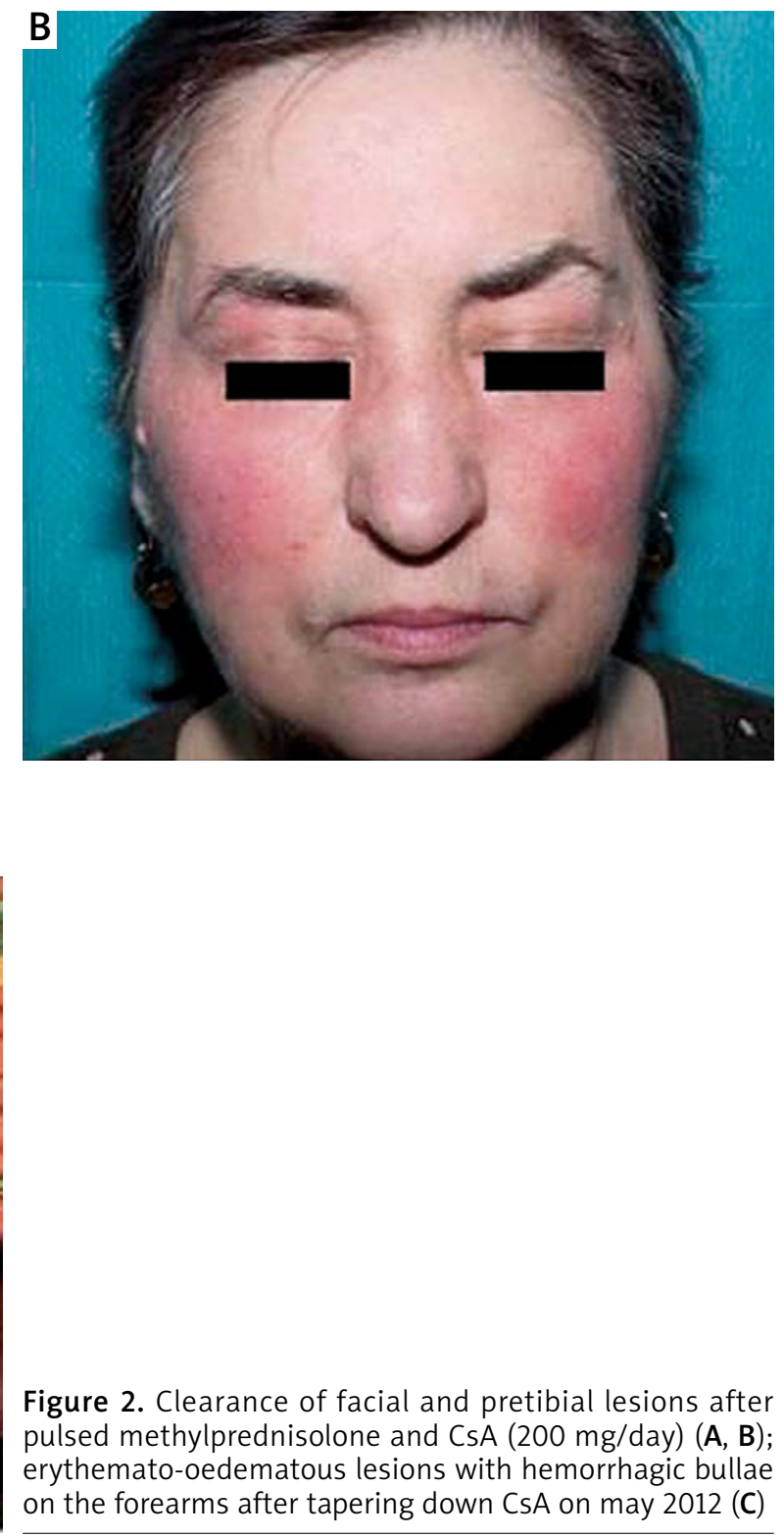
Currently (July 2012), the patient is clear from skin lesions and continues taking CsA (150 mg/day) and prednisone (40 mg/20 mg). The patient remains under the supervision of the Haematology-Oncology Clinic, as she requires blood testing and blood transfusions periodically.

\section{Discussion}

The reported case of atypical bullous PG presented the diagnostic and therapeutic difficulties. Initially, the patient was wrongly diagnosed with ophthalmic zoster due to unilateral erythemato-infiltrative lesions with exudation confined to the right periorbital area and treated unsuccessfully with acyclovir. Afterwards, involvement of the analogous left-sided area was recognized as preseptal cellulitis and empiric systemic antibiotic therapy was introduced without significant improvement. Finally, hemorrhagic bullae with necrosis enabled the correct diagnosis of bullous PG. The authors find this case atypical due to the unusual presentation of skin lesions in the form of multiple, multifocal hemorrhagic bullae with necrosis and association with pancytopenia of unknown origin.

Bullous PG was first described by Perry and Winkelmann in 1972 as hemorrhagic bullae rapidly evolving to superficial ulcerations [5]. The feature differentiating bullous PG from classic PG is the superficial ulcer with bullous borders in the former as opposed to deep, destructive lesions with undermined borders seen in the classic variant [6]. The sites of predilection include the face, hands and legs [7]. Bullous PG is frequently associated with systemic disorders such as lymphoproliferative diseases [1, 5, 6, 8-14], inflammatory bowel disease [5, 15-17], rheumatoid arthritis [5] and Klinefelter's syndrome $[5,18]$. According to Su et al., bullous PG is associated with haematological malignancy in up to $70 \%$ of cases [19]. Sakiyama et al. reviewed 38 cases of bullous PG and demonstrated that the most commonly associated haematological disorders (65.8\%) included acute myeloblastic leukaemia (44.7\%) and chronic myelogenous leukaemia (10.5\%) (Table 1) [5]. Only in 7 cases (18.4\%) there were no underlying diseases diagnosed [5]. Moreover, Sakiyama et al. reported that females were predominantly affected (65.8\%) and the mean age was 51.6 years [5]. Bullous PG typically responds to treatment of the underlying disorder. Jacobs et al. observed that chemotherapy for the underlying malignant disorder often resulted in clearance of PG lesions [6]. In refractory cases or cases not associated with any disease, systemic therapies as in the classical PG variant should be introduced [6].

In the present case, the patient was under the supervision of the Haematology-Oncology Clinic for repeated declines in haematopoietic parameters. According to Koester et al., any patient with neutrophilic dermatosis and unexplained anaemia should undergo appropriate examinations to exclude leukaemia or myelodysplasia
Table 1. Diseases most commonly associated with bullous PG [4]

\begin{tabular}{lcc}
\hline \multicolumn{1}{c}{ Associated disease } & $\begin{array}{c}\text { Number } \\
\text { of patients }\end{array}$ & $\begin{array}{c}\text { Percentage } \\
\text { of patients }\end{array}$ \\
\hline Acute myeloblastic leukaemia & 17 & 44.7 \\
\hline Chronic myelogenous leukaemia & 4 & 10.5 \\
\hline Myelodysplastic syndrome & 2 & 5.3 \\
\hline Multiple myeloma & 1 & 2.6 \\
\hline Myeloid metaplasia & 1 & 2.6 \\
\hline Inflammatory bowel disease & 4 & 10.5 \\
\hline Rheumatoid arthritis & 1 & 2.6 \\
\hline Klinefelter's syndrome & 1 & 2.6 \\
\hline No associated disease & 7 & 18.4 \\
\hline
\end{tabular}

[20]. In the case described, repeated bone marrow biopsies with immunophenotyping excluded proliferative haematological disorders, whereas PET revealed no features of neoplastic disease. Thus, the final diagnosis was not established. However, the features of dysplasia in the most recent bone marrow examination suggest that possible development of myelodysplastic syndrome should be considered.

\section{Conclusions}

Our case posed diagnostic as well as therapeutic difficulties. Therapy with methylprednisolone, intravenous infusions of immunoglobulins and PRBC did not satisfactorily improve blood cell count parameters. Moreover, tapering the dose of CsA resulted in the development of new skin lesions. At present, the patient requires periodic transfusions of PRBC and PLT, as well as haematological observation with regular bone marrow examinations to confirm or exclude diseases of the haematopoietic system.

\section{Conflict of interest}

The authors declare no conflict of interest.

\section{References}

1. Ghazal PA, Herberger K, Schaller J, et al. Associated factors and comorbidities in patients with pyoderma gangrenosum in Germany: a retrospective multicentric analysis in 259 patients. Orphanet J RarDis 2013; 8: 136-44.

2. Goeckerman WH, O'Leary PA. Pyoderma (ecthyma) gangrenosum. Clinical and experimental observation in five cases. Arch Dermatol Syphilol 1930; 221: 655-80.

3. Torzecka JD, Kondras K, Dziankowska-Bartkowiak B, et al. Pyoderma gangrenosum - an interdisciplinary diagnostic problem [Polish]. Pol Merk Lek 2008; 139: 34-7.

4. Maj J, Maciąg H, Barancewicz-Łosek M, et al. Pyoderma gangrenosum resembling Paget's disease [Polish]. Contemp Onkol (Poznań) 2006; 9: 464-7. 
5. Sakiyama M, Kobayashi T, Nagata Y, et al. Bullous pyodermia gangrenosum: a case report and review of the published work. J Dermatol 2012; 39: 1-6.

6. Jackobs P, Palmer S, Gordon-Smith EC. Pyoderma gangrenosum in myelodyspalsia and acute leukaemia. Postgrad Med J 1985; 61: 689-94.

7. Bhat RM. Pyoderma gangrenosum: an update. Indian Dermatol Online I 2012; 1: 7-13.

8. Batista MD, Ikino JK, Michalny NS, et al. Bullous pyoderma gangrenosum and myelodysplastic syndrome. An Bras Dermatol 2006; 81: 309-12.

9. Beele H, Verhaeghe E, Stockman A, et al. Pyoderma gangrenosum as an early revelator of acute leukemia. Dermatology 2000; 200: 176-8.

10. Fox LP, Geyer AS, Husain S, et al. Bullous pyoderma gangrenosum as the presenting sign of fatal acute myelogenous leukemia. Leuk Lymphoma 2006; 1: 147-50.

11. Wiseman DH, Hunter HJA, Dennis M. Bullous pyoderma gangrenosum in acute myeloid leukaemia. Eur J Haematol 2007; 79: 91-2.

12. Rafael MR, Fernandes CM, Machado JM, et al. Pyoderma gangrenosum or leukaemia cutis. Eur Acad Dermatol Venereol 2003; 17: 449-51.

13. Hay CRM, Messenger AG, Cotton DWK, et al. Atypical bullous pyoderma gangrenosum associated with myeloid malignancies. J Clin Pathol 1987; 40: 387-92.

14. Lewerin C, Mobacken H, Nilsson-Ehle H, et al. Bullous pyoderma gangrenosum in a patient with myelodysplastic syndrome during granulocyte colony-stimulating factor therapy. Leu Lymphoma 1997; 26: 629-32.

15. LalNaik W, Singh G, Kumar L, et al. Bullous PG associated with ulcerative colitis. Indian I Dermatol Venereol Leprol 2008; 1: 68-70

16. Akay N, Boyvat A, Heper AO, et al. Bechet's disease-like presentation of bullous PG associated with Crohn's disease. Clin Exp Dermatol 2006; 3: 384-6

17. Singh G, Sethi A, Okade R, et al. Bullous pyoderma gangrenosum: a presentation of childhood Bechet's disease. Int J Dermatol 2005; 44: 257-8.

18. Marzano AV, Trevisan V, Galloni C. Fatal bullous pyoderma gangrenosum in a patient with Klinefelter's syndrome. Acta Derm Venereol 2008; 88: 158-9.

19. Su WP, Davis MDP, Weening RH, et al. Pyoderma gangrenosum: clinicopathologic correlation and proposed diagnostic criteria. Int J Dermatol 2004; 43: 790-800.

20. Koester G, Tarnowe A, Levisohn D, et al. Bullous pyoderma gangrenosum. J Am Acad Dermatol 1997; 29: 875-8. 